\title{
COVID-19, pandemia y acúfeno
}

\section{COVID-19, pandemic and tinnitus}

\author{
María Angélica Velasco O. ${ }^{1}$, Laura Julia Jardines S. ${ }^{1}$, Idalia Mercado H. ${ }^{1}$, \\ María Vanessa Castañeda M. ${ }^{1}$, Ana Luisa Lino G. $^{2}$
}

\section{Resumen}

El acúfeno es un síntoma que puede presentarse aislado o en diferentes patologías. Se describe como la percepción de un sonido o percepción auditiva fantasma no producida por una vibración o estímulo acústico externo, que es inaudible para el entorno. La COVID-19 causada por el virus SARS-CoV-2, es una enfermedad que ha mostrado diversidad en su expresión, severidad, síntomas y secuelas. El acúfeno es una condición relacionada con el sistema auditivo que ha sido estudiada durante la pandemia por COVID-19 y se ha podido observar un incremento en la incidencia y/o exacerbación de la percepción de este síntoma, en donde se involucran múltiples factores que se vuelven importantes de considerar.

Palabras clave: COVID-19, pandemia, acúfeno.

\begin{abstract}
Tinnitus is a symptom that can occur in isolation or in different pathologies. It is described as the perception of a sound, or as a phantom auditory perception that is not produced by a vibration or external acoustic stimulus, which is inaudible to the environment. COVID-19 caused by the SARS-CoV-2 virus, is a disease that has shown diversity in its expression, severity, symptoms and sequelae. Tinnitus is a condition related to the auditory system that has been studied during the COVID-19 pandemic, and an increase in the incidence and/or exacerbation of the perception of this symptom has been observed, involving multiple factors that become important to consider.
\end{abstract}

Keywords: COVID-19, pandemic, tinnitus.
'Subdirección de Audiología, Foniatría y Patología del Lenguaje. División de Terapia en Comunicación Humana. Instituto Nacional de Rehabilitación "Luis Guillermo Ibarra Ibarra". Ciudad de México, México.

²Subdirección de Investigación Biomédica. División de Neurociencias. Área de Neurociencia clínica, Instituto Nacional de Rehabilitación "Luis Guillermo Ibarra Ibarra". Ciudad de México, México.

Los autores declaran no tener conflictos de interés.

Recibido el 13 de septiembre de 2021. Aceptado el 24 de octubre de 2021

Correspondencia:

Ana Luisa Lino G.

Calzada México Xochimilco No. 289.

Colonia Arenal de Guadalupe. C.P.14389.

Ciudad de México, México.

Email: ana_onil@yahoo.com.mx

\section{Introducción}

A finales de 2019, específicamente en el mes de diciembre, dio la vuelta al mundo una noticia que iniciaría una serie de eventos que afectarían al todo el planeta. Se trataba de la presencia de los primeros casos en China de un nuevo coronavirus denominado SARS-CoV-2, causante del síndrome respiratorio agudo grave de tipo 2 o enfermedad por coronavirus de 2019 (COVID-19) ${ }^{1}$. Se trata de un virus de origen zoonótico que ha resultado ser altamente contagioso entre los seres humanos ${ }^{2}$, situación que llevó al mundo entero por exhortación de organizaciones internacionales como la Organización Mundial de la Salud y la Organización
Panamericana de la Salud a realizar recomendaciones con la intención de disminuir el número de contagios, evitar una alta demanda de los servicios hospitalarios, la escases de insumos y de personal médico calificado, ya que en muchos casos, los pacientes requerían de hospitalización y/o atención en unidades de cuidados intensivos con ventilación mecánica. Con el paso de los días surgió información diversa, resultado de hallazgos realizados por personal médico de diferentes especialidades y también por los propios pacientes, ya que se trataba de una enfermedad que al parecer podía expresarse de diferentes formas en cuestión de severidad, síntomas y secuelas. Una de las tantas condiciones reportadas durante este 
tiempo pandémico se relaciona con el sistema audio-vestibular, específicamente el síntoma de tinnitus o acúfeno.

El objetivo de la presente revisión es describir las experiencias reportadas en la literatura científica publicada en 2020 y principios de 2021 con relación a la enfermedad de COVID-19, la pandemia por COVID-19 y el acúfeno.

\section{Acúfeno}

El término tinnitus tiene su origen en el latín "tinnire" y su significado es tintineo o campañilleo $^{3,4}$, así mismo, la palabra acúfeno procede del griego y hace referencia a un sonido que aparece ${ }^{4}$. El tinnitus o acúfeno es un síntoma que puede presentarse aislado o en diferentes patologías. Gracias al avance en la investigación y al desarrollo tecnológico se ha podido establecer que se trata de un trastorno con participación de la vía auditiva y de diferentes áreas del sistema nervioso central que están relacionadas con la audición ${ }^{4,5}$. Se define como la percepción de un sonido o percepción auditiva fantasma no generada por una vibración o estímulo acústico externo, que es inaudible para el entorno ${ }^{3,4,6}$; en otras palabras, se trata de un fenómeno psicosensorial experimentado en la corteza auditiva ${ }^{4}$.

Según el National Institute on Deafness and other Communication Disorders, el acúfeno es un timbre o silbido en los oídos, que puede sonar como rugido, chasquido, seseo o zumbido, percibido con una intensidad suave o fuerte $\mathrm{y}$ un tono agudo o grave por uno o ambos oídos ${ }^{7}$, así mismo, la American Speech Language Hearing Association, se refiere al acúfeno como un "zumbido en los oídos" presente aún en ausencia de ruido y agrega que puede ser percibido como un ronquido, latido, silbido, repique, chirrido o chasquido ${ }^{8}$. La prevalencia del acúfeno reportada en la literatura científica se ubica de $6,6 \%$ a $21,5 \%$ en población adul$\mathrm{ta}^{3,6,9,10} \mathrm{y}$ se destaca un incremento importante después de los 55 años $^{11}$.

\section{Acúfeno durante la pandemia por COVID-19}

En la Tabla 1, se describen 10 estudios publicados durante 2020 y principios de 2021 (un caso clínico y nueve estudios empíricos) que reportan sus hallazgos en la literatura científica. En el caso de los estudios empíricos, cuatro se realizaron en pacientes con acúfeno diagnosticado previamente a la pandemia de COVID-19, a quienes se les dio seguimiento con el objetivo de evaluar el impacto de la pandemia en la expresión de este síntoma; los restantes cinco estudios, se realizaron con pacientes positivos a COVID-19, confirmado con prueba de reacción en cadena de la polimerasa con transcripción inversa (RT-PCR), o que se encontraban en cuarentena por presentar síntomas de COVID-19 y a quienes se les realizaron evaluaciones otorrinolaringológicas que reportaban acúfeno, por último, el caso clínico corresponde a una paciente positiva a COVID-19. Con respecto a los países en los que se llevaron a cabo los estudios, tres (30\%) se realizaron en Italia, dos (20\%) en Reino Unido, dos (20\%) en Turquía y uno en Alemania, Qatar y China respectivamente.

A continuación, se describen los hallazgos realizados. En primera instancia, con respecto a los estudios llevados a cabo en pacientes con diagnóstico previo de acúfeno, Schlee y cols. ${ }^{12}$ evaluaron a 122 pacientes con la intención de medir el impacto de la pandemia en el estrés y la angustia, así como, su relación con el acúfeno, sugieren que su estudio aporta información con respecto a la influencia ambiental sobre este síntoma auditivo. Destacan que los pacientes con diagnóstico previo de acúfeno que percibieron frustración, estrés y nerviosismo durante la pandemia, reportaron un incremento de la angustia relacionada con el acúfeno, así mismo, sugieren que una mayor puntuación de neurosis refleja un considerable empeoramiento del síntoma. Beukes y cols. ${ }^{13}$ realizaron una encuesta a 3103 personas de 48 países; los autores encontraron que el acúfeno se calificó como más molesto (32\%) y más estable $(67 \%)$ durante la pandemia, sin embargo, destacan que fueron las mujeres y los menores de 50 años quienes reportaron que el acúfeno fue significativamente más molesto. Por otra parte, en aquellos participantes con diagnóstico previo de acúfeno que también presentaron síntomas de COVID-19, el 54\% refirió presentar acúfeno estable y $40 \%$ acúfeno agravado. Los pacientes que informaron alguno de los siguientes factores: autoaislamiento, aumento de nivel de estrés por implementación de actividades laborales y escolares de sus hijos o de 
Tabla 1. Hallazgos sobre acúfeno durante la pandemia de COVID-19

\begin{tabular}{|c|c|c|c|c|c|}
\hline Autores & $\begin{array}{l}\text { País } \\
\text { Año }\end{array}$ & Objetivo & Muestra & Método & Resultados \\
\hline $\begin{array}{l}\text { Schlee y } \\
\text { cols. }^{12}\end{array}$ & $\begin{array}{l}\text { Alemania } \\
2020\end{array}$ & $\begin{array}{l}\text { Impacto de la } \\
\text { pandemia en estrés } \\
\text { percibido y la } \\
\text { angustia relacionada } \\
\text { con el acúfeno }\end{array}$ & $\begin{array}{l}122 \text { pacientes con } \\
\text { acúfeno } \\
\text { Edad media } 54 \text { años }\end{array}$ & $\begin{array}{l}\text { - Evaluación de } \\
\text { acúfeno y depresión } \\
\text { - Evaluación de } \\
\text { agobio }\end{array}$ & $\begin{array}{l}\text { Frustración, estrés y } \\
\text { nerviosismo } \\
\text { Aumentó de angustia } \\
\text { relacionada con } \\
\text { acúfeno }\end{array}$ \\
\hline $\begin{array}{l}\text { Beukes y } \\
\text { cols. }^{13}\end{array}$ & $\begin{array}{l}\text { Reino } \\
\text { Unido } \\
2020\end{array}$ & $\begin{array}{l}\text { Impacto de la } \\
\text { pandemia en } \\
\text { personas con acúfeno } \\
\text { e identificar factores } \\
\text { mediadores }\end{array}$ & $\begin{array}{l}3.103 \text { pacientes } \\
\text { de } 48 \text { países con } \\
\text { acúfeno } \\
\text { Media de edad } 58 \\
\text { años }\end{array}$ & $\begin{array}{l}\text { - Información } \\
\text { demográfica, } \\
\text { duración y gravedad } \\
\text { de acúfeno, efectos } \\
\text { de confinamiento }\end{array}$ & $\begin{array}{l}\text { Acúfeno más molesto } \\
\text { durante la pandemia. } \\
\text { Aumento de ansiedad } \\
\text { afectó negativamente } \\
\text { al acúfeno }\end{array}$ \\
\hline $\begin{array}{l}\text { Xia y } \\
\text { cols. }{ }^{14}\end{array}$ & $\begin{array}{l}\text { China } \\
2020\end{array}$ & $\begin{array}{l}\text { Investigar si la } \\
\text { ansiedad asociada } \\
\text { con COVID-19 es un } \\
\text { factor promotor del } \\
\text { acúfeno }\end{array}$ & $\begin{array}{l}89 \text { pacientes (2019) } \\
99 \text { pacientes }(2020) \\
\text { con acúfeno }\end{array}$ & $\begin{array}{l}\text { - Datos clínicos } \\
\text { - Estudios } \\
\text { audiológicos } \\
\text { - Evaluación de } \\
\text { acúfeno }\end{array}$ & $\begin{array}{l}\text { Incremento de casos } \\
(11,2 \%) \text { en } 2020 \text { y } \\
\text { ansiedad más alta } \\
\text { relacionada con la } \\
\text { pandemia }\end{array}$ \\
\hline $\begin{array}{l}\text { Anzivino } \\
\text { y cols. }{ }^{15}\end{array}$ & $\begin{array}{l}\text { Italia } \\
2020\end{array}$ & $\begin{array}{l}\text { Grado de } \\
\text { discapacidad } \\
\text { autopercibida durante } \\
\text { el confinamiento } \\
\text { en pacientes con } \\
\text { acúfeno crónico }\end{array}$ & $\begin{array}{l}16 \text { pacientes con } \\
\text { acúfeno }\end{array}$ & $\begin{array}{l}\text {-Evaluación de } \\
\text { acúfeno }\end{array}$ & $\begin{array}{l}\text { Grado de } \\
\text { discapacidad por } \\
\text { acúfeno aumentó en } \\
12 \text { de } 16 \text { pacientes } \\
(75 \%)\end{array}$ \\
\hline $\begin{array}{l}\text { Micarelli } \\
\text { y cols. }{ }^{16}\end{array}$ & $\begin{array}{l}\text { Italia } \\
2020\end{array}$ & $\begin{array}{l}\text { Síntomas generales } \\
\text { y ORL percibidos de } \\
\text { forma somática por } \\
\text { COVID-19 en sujetos } \\
\text { en cuarentena }\end{array}$ & $\begin{array}{l}1.380 \text { pacientes en } \\
\text { cuarentena } \\
543 \text { hombres } \\
837 \text { mujeres }\end{array}$ & $\begin{array}{l}\text { Impacto psicológico, } \\
\text { salud mental, } \\
\text { percepción de } \\
\text { la información } \\
\text { COVID-19 }\end{array}$ & $\begin{array}{l}\text { El 10,4\% refirió } \\
\text { acúfeno }\end{array}$ \\
\hline $\begin{array}{l}\text { Korkmaz } \\
\text { y cols. }{ }^{17}\end{array}$ & $\begin{array}{l}\text { Turquía } \\
2021\end{array}$ & $\begin{array}{l}\text { Sintomatología } \\
\text { ORL de pacientes } \\
\text { COVID-19 con } \\
\text { seguimiento en la } \\
\text { clínica de ORL }\end{array}$ & $\begin{array}{l}116 \text { pacientes con } \\
\text { COVID-19 } \\
\text { Edad media 57,2 } \\
\text { años }\end{array}$ & $\begin{array}{l}\text { Datos demográficos } \\
\text { enfermedades } \\
\text { previas, medicación y } \\
\text { hospitalización }\end{array}$ & $\begin{array}{l}\text { La tasa de acúfeno } \\
\text { fue de } 11,2 \% \text {. } \\
\text { Significativo en } \\
\text { menores de } 60 \text { años. }\end{array}$ \\
\hline Elibol $^{18}$ & $\begin{array}{l}\text { Turquía } \\
2020\end{array}$ & $\begin{array}{l}\text { Frecuencia de } \\
\text { síntomas ORL en } \\
\text { pacientes con } \\
\text { COVID-19 con } \\
\text { respecto a edad y } \\
\text { sexo de los pacientes }\end{array}$ & $\begin{array}{l}155 \text { pacientes } \\
91 \text { mujeres } \\
64 \text { hombres } \\
\text { Media de edad 36,3 } \\
\text { años }\end{array}$ & $\begin{array}{l}5 \text { grupos } \\
\text { Cuestionario sobre } \\
\text { síntomas }\end{array}$ & $\begin{array}{l}\text { El síntoma ORL } \\
\text { menos frecuente fue } \\
\text { el acúfeno }(1,2 \%)\end{array}$ \\
\hline $\begin{array}{l}\text { Munro y } \\
\text { cols. }^{19}\end{array}$ & $\begin{array}{l}\text { Reino } \\
\text { Unido } \\
2020\end{array}$ & $\begin{array}{l}\text { Investigar síntomas } \\
\text { ORL en pacientes con } \\
\text { COVID-19 }\end{array}$ & $\begin{array}{l}138 \text { pacientes con } \\
\text { síntomas graves de } \\
\text { COVID-19 }\end{array}$ & $\begin{array}{l}\text { Historia clínica } \\
\text { Cuestionario sobre } \\
\text { audición y acúfeno }\end{array}$ & $\begin{array}{l}16 \text { pacientes } \\
\text { reportaron acúfeno } \\
\text { desde el diagnóstico }\end{array}$ \\
\hline $\begin{array}{l}\text { Viola y } \\
\text { cols. }^{20}\end{array}$ & $\begin{array}{l}\text { Italia } \\
2020\end{array}$ & $\begin{array}{l}\text { Prevalencia de } \\
\text { acúfeno y trastorno } \\
\text { del equilibrio en } \\
\text { pacientes con } \\
\text { COVID-19 }\end{array}$ & $\begin{array}{l}185 \text { pacientes } \\
99 \text { hombres } \\
86 \text { mujeres } \\
\text { Edad media de 52,1 } \\
\text { años }\end{array}$ & $\begin{array}{l}\text { Cuestionario } \\
\text { sobre presencia y } \\
\text { características del } \\
\text { acúfeno }\end{array}$ & $\begin{array}{l}43 \text { pacientes } \\
\text { refirieron acúfeno. } \\
14 \text { informaron } \\
\text { acúfeno y trastorno } \\
\text { del equilibrio }\end{array}$ \\
\hline $\begin{array}{l}\text { Chirakkal } \\
\text { y cols. }{ }^{21}\end{array}$ & $\begin{array}{l}\text { Qatar } \\
2021\end{array}$ & & $\begin{array}{l}\text { Paciente mujer de } 35 \\
\text { años con antecedente } \\
\text { de COVID-19 } \\
\text { sín síntomas de } \\
\text { neumonía }\end{array}$ & $\begin{array}{l}\text { Evaluación } \\
\text { audiológica detallada }\end{array}$ & $\begin{array}{l}\text { Acúfeno a } 4 \mathrm{kHz} \text { a } \\
10 \mathrm{~dB} \\
\text { Daño en células } \\
\text { ciliadas externas }\end{array}$ \\
\hline
\end{tabular}


ellos mismos en el hogar, incremento de tareas domésticas, sensación de soledad, dificultad para dormir, tristeza, depresión, ansiedad, irritabilidad, incremento en el consumo de cafeína o alcohol, preocupaciones financieras o que realizaron menos ejercicio físico reportaron acúfeno significativamente más molesto; de igual forma, el uso continuo de auriculares y la participación constante en videoconferencias y llamadas telefónicas, así como, la tensión en el cuello por pasar una gran parte del día usando computadoras impactaron de forma negativa la percepción del acúfeno, de tal manera que, los autores concluyen que el incremento de la ansiedad afectó de forma significativa.

Xia y cols. ${ }^{14}$ compararon las características clínicas del acúfeno entre pacientes diagnosticados en el mismo periodo (marzo-abril) de 2019 y de 2020; estos últimos bajo presión pandémica. Los autores encontraron un incremento de $11,2 \%$ en el diagnóstico de acúfeno en el periodo del 2020; el 74,7\% de los diagnosticados presentaron el criterio de ansiedad significativamente más alto y las puntuaciones obtenidas por estos pacientes en el tinnitus handicap inventory fueron significativamente más altas, lo cual, a decir de los autores podría estar relacionado con el aumento de la ansiedad. Por otra parte, sugieren que el incremento de la ansiedad en los pacientes de 2020 está relacionado con la pandemia por COVID-19, esto, al verse afectada su forma de vida. Concluyen que el estudio proporciona evidencia sobre el efecto inductor de la ansiedad sobre el acúfeno.

Anzivino y cols. ${ }^{15}$ realizaron un estudio cuyos participantes eran pacientes con acúfeno crónico, la intención fue medir el grado de discapacidad autopercibida producida por este síntoma, encontrando que $62,5 \%$ reportaron discapacidad moderada, $18,7 \%$ grave, $12,5 \%$ catastrófica y 6,2\% leve. El grado autopercibido de discapacidad aumentó en el 75\% de los pacientes. Los autores sugieren que la propensión a preocuparse y el estrés por la pandemia podrían incluirse como factores de riesgo para el empeoramiento del acúfeno.

Con respecto a los estudios realizados en pacientes positivos a COVID-19 o que se encontraban en cuarentena por presentar síntomas, Micarelli y cols. ${ }^{16}$ reportaron que de 1.380 participantes en situación de cuarentena por presentar síntomas de COVID-19, 144 $(10,4 \%)$ refirieron acúfeno, Korkmaz y cols. ${ }^{17}$ estudiaron a 116 pacientes con COVID-19 con la intención de evaluar síntomas otorrinolaringológicos, encontrando como parte de los síntomas audio-vestibulares una tasa de acúfeno de 11,2\%; al agrupar a los pacientes con respecto a la edad se encontró que el acúfeno, fue estadísticamente significativo en los pacientes menores de 60 años. Elibol ${ }^{18}$, realizó un estudio con 155 pacientes positivos a COVID-19 evaluando también la presencia de síntomas otorrinolaringológicos; encontró que $2(1,2 \%)$ pacientes reportaron la presencia de acúfeno, ubicándose como el síntoma menos frecuente. Munro y cols. ${ }^{19}$ desarrollaron un estudio en el que participaron 138 pacientes con síntomas graves de COVID-19 de los cuales ocho informaron presencia de acúfeno, uno refirió acúfeno unilateral izquierdo con sensación de presión auditiva y uno más, informó la presencia de acúfeno que desapareció con el paso del tiempo, a partir del diagnóstico de la enfermedad por coronavirus. Viola y cols. ${ }^{20}$ consideraron un total de 185 pacientes entre 30 a 60 días después de ser diagnosticados con COVID-19, con la finalidad de investigar la prevalencia de varios trastornos entre ellos el acúfeno, a través de un cuestionario en línea, encontrando este síntoma en 43 (23,2\%) pacientes, correspondiente a 28 hombres y 15 mujeres, de los cuales, 17 reportaron acúfeno recurrente (aparecía y desaparecía durante el día), 10 refirieron acúfeno ocasional (episódico, esporádico), 7 acúfeno fluctuante durante el día (continuo con intensidad variable), 4 acúfeno persistente (siempre presente, durante el día y la noche), 3 acúfeno pulsátil (sincrónico con los latidos del corazón), 2 acúfeno continuo (siempre presente, a la misma intensidad, dificultando el sueño), así mismo, 14 pacientes informaron acúfeno junto con trastorno del equilibrio después del diagnóstico. Por último, con respecto al caso clínico encontrado en la literatura, se destaca que se trata de una paciente de 35 años con antecedente de COVID-19 quien acudió a consulta con sensación de acúfeno y disminución de sensibilidad auditiva en el oído izquierdo. Se refiere que durante la enfermedad recibió tratamiento conservador en su domicilio por no presentar indicios de neumonía, los síntomas audiológicos apare- 
cieron después del diagnóstico y persistieron después de la recuperación. Se realizó acufenometría, observándose acúfeno en la frecuencia de $4 \mathrm{kHz}$ a una intensidad de $10 \mathrm{~dB}^{21}$.

\section{Discusión}

La COVID-19 se ha caracterizado no únicamente por afectar al sistema respiratorio. Con el paso del tiempo, gracias a diferentes estudios científicos, se han podido conocer diversas afecciones ligadas con los diferentes sistemas del cuerpo humano, que pueden aparecer en el momento de instauración de la enfermedad e incluso continuar o manifestarse después de la recuperación del paciente, de esta manera, se ha podido detectar que el sistema auditivo también se puede ver afectado, y en particular con respecto al síntoma de acúfeno.

Considerando los puntos de vista de los autores de los artículos revisados nos parece importante destacar que, en el caso del acúfeno encontramos dos situaciones en las que ha sido posible estudiarlo, por un lado, en los pacientes positivos a la enfermedad de COVID-19 y por otro, en pacientes con diagnóstico de acúfeno previo a la pandemia.

En lo que respecta a los pacientes con diagnóstico previo encontramos algunas cuestiones que vale la pena destacar, como el hecho de que, asuntos principalmente de índole emocional desencadenados o exacerbados por la pandemia de COVID-19 influyen, promueven o empeoran este síntoma, destacándose a decir de los autores factores como frustración, estrés, nerviosismo, incremento de angustia ${ }^{12,13}$, de ansiedad ${ }^{14} y$ preocupación por la pandemia ${ }^{15}$; en el caso específico de los pacientes con diagnóstico previo de acúfeno y que enfermaron de COVID-19, los autores destacan nuevamente aspectos emocionales entre ellos, soledad, tristeza, depresión, irritabilidad y preocupaciones financieras como promotores de acúfeno significativamente más molesto; todos estos reportes concuerdan con lo descrito previamente por diferentes autores que refieren a factores como la depresión, la ansiedad y el estrés estrechamente relacionados con la aparición y la gravedad del acúfeno ${ }^{6,9,11,22,23}$. Con respecto a esto, Baguley ${ }^{24}$ refiere que en los casos en los que el acúfeno se relaciona como refuerzo emocional negativo (miedo, ansiedad o tensión), el sistema límbico y la activación autónoma provocan que la actividad se incremente y la percepción del acúfeno persista, así mismo, Sáez-Jiménez y Herráiz-Puchol ${ }^{25}$ argumentan que las conexiones con el sistema límbico y con la corteza prefrontal son responsables de la fuerte influencia del estado de ánimo sobre la intensidad del acúfeno.

Por otra parte, también se reportaron otros factores, relacionados con la modificación en el estilo de vida durante la pandemia, como son, problemas para dormir, incremento de tareas domésticas, de consumo de cafeína y alcohol, diminución de ejercicio físico, uso continuo de auriculares y tensión en el cuello por uso prolongado de computadoras ${ }^{13}$. Previamente diferentes investigadores han reportado la asociación entre el consumo de alcohol y tabaco con el acúfeno ${ }^{9,11}$. Con respecto al uso continuo de auriculares se ha reportado también como un factor de riesgo ${ }^{11}$; por otra parte, pacientes con dolor de cuello (en este caso posiblemente por el uso prolongado de computadoras) $)^{3,23} \mathrm{y}$ el abuso de cafeína, se contemplan como factores que desencadenan o agravan el acúfeno ${ }^{3}$. Otro hallazgo que se destaca, tiene que ver con el reporte de acúfeno significativamente más molesto en mujeres y en menores de 50 años ${ }^{13}$. A este respecto la literatura científica hace referencia a que el acúfeno suele ser más frecuente en el sexo masculino, sin embargo, se aclara que esto tiene que ver con el hecho de que en muchos ambientes laborales, en donde es más frecuente la participación de los hombres, los niveles de ruido son elevados o los arriesga a una mayor exposición a ruido ${ }^{11}$; por otra parte, también encontramos estudios en donde se hace referencia a mayor presencia de este síntoma en mujeres, datos que concuerda con lo reportado por Beukes ${ }^{13}$, y a decir de Valente y cols. ${ }^{3}$ se debe posiblemente a la conocida mayor prevalencia de trastornos psicológicos en el sexo femenino, o al estrés asociado con este género en diferentes países ${ }^{11}$, de tal manera, que considerando la situación de la mujeres durante la pandemia, es posible que los niveles de ansiedad y depresión por la sobrecarga de actividades habituales, ya sean en el hogar, en el trabajo o ambos hayan sido un factor detonante. Por otro lado, el acúfeno fue significativamente más molesto en lo menores 
de 50 años ${ }^{13} y$ significativamente más frecuente en menores de 60 años $^{17}$, dato que contrasta con lo reportado en la literatura, en donde se hace referencia a que este síntoma suele ser más frecuente conforme incrementa la edad y en donde suele acompañarse de una disminución de la audición ${ }^{23,24,26}$, como lo muestra el estudio realizado en Corea del Sur en donde obtuvieron una prevalencia mayor a $30 \%$ en mayores de 70 años y $36 \%$ a partir de los $85^{11}$, por tanto, considerando el hecho de que las mujeres y los más jóvenes percibieron como más molesto el acúfeno durante la pandemia, Beukes y cols. ${ }^{13}$, sugieren que podría atribuirse a mayores cambios en el estilo de vida de estos grupos durante esta contingencia, como serían el trabajo en casa, mayor cuidado de los niños e incremento de las responsabilidades domésticas, e incluso se podría contemplar la posibilidad de que factores como el uso excesivo de auriculares para tomar clases en línea, videoconferencias y llamadas telefónicas que ha sido tan necesario durante esta pandemia estén relacionados, aunque es posible también, que factores emocionales asociados con la enfermedad de COVID-19, el confinamiento y el distanciamiento social también hayan contribuido.

Considerando, por un lado, el aumento de la discapacidad autopercibida por acúfeno, y por otro, la sensación del acufeno como más molesto, se sugiere la posibilidad de que, durante el confinamiento, la ausencia de sonidos ambientales de enmascaramiento que son habituales en la vida cotidiana podría haber exacerbado la percepción del acúfeno ${ }^{1}$.

En los estudios realizado en pacientes positivos a COVID-19 que reportaron la presencia de acúfeno después de haber sido diagnosticados, se encontró una prevalencia que varía de $1,2 \%$ al 23,2\% ${ }^{16-18,20}$, datos que concuerdan con la literatura revisada, en donde también se observa un amplio rango $(6,6 \% \text { a } 21,5 \%)^{3,6,9,10}$, y en donde es posible que los datos respondan a factores relacionados con el tamaño de la muestra y los criterios de inclusión considerados.

Analizando los resultados reportados en los pacientes positivos a COVID-19 que presentaron acúfeno posterior al diagnóstico de la enfermedad, Viola y cols. ${ }^{20}$ hacen referencia a que algunas infecciones virales causan hipoa- cusia, como consecuencia de daño al tronco encefálico auditivo y a las células del oído interno; se contempla también que los síntomas neurológicos relacionados con el coronavirus como es el caso del acúfeno pueden atribuirse a la capacidad neurotrófica y neuroinvasiva típica de algunos coronavirus, así mismo, sugieren que, los efectos del SARS-CoV-2 sobre el tejido neuronal podrían deberse a una infección directa del sistema nervioso central o estar relacionados con un daño vascular causado por vasculitis o vasculopatía, ya que ha surgido evidencia de que pacientes con COVID-19 han presentado signos de hipercoagulabilidad o vasculitis, y está demostrado también, que las estructuras del oído interno son susceptibles a la isquemia, como consecuencia de sus características de vasculatura terminal y el alto requerimiento de energía, de igual manera, Chirakkal y cols. ${ }^{21}$ argumentan que la infección por COVID-19 tiene efectos nocivos sobre las células ciliadas externas de la cóclea y que el daño del sistema auditivo suele ser intracoclear; destacan también, el hecho de que algunos virus pueden afectar el tronco cerebral auditivo.

De acuerdo con algunos autores, se ha considerado que no está claro cuáles casos de acúfeno, pueden atribuirse directamente al SARS-CoV-2 o bien, relacionarse con muchas otras causas posibles de pérdida de audición asociadas con cuidados críticos, como, el uso de medicamentos ototóxicos ${ }^{19}$ (salicilatos, quinina, antibióticos aminoglucósidos y fármacos a base de platino) ${ }^{9}$ administrados de forma local o sistémica, infecciones, trastornos vasculares y enfermedades autoinmunes, así mismo, sugieren que la ansiedad asociada con el diagnóstico y la recuperación de COVID-19 puede contribuir a este síntoma ${ }^{19}$.

\section{Conclusión}

En el marco de la pandemia por COVID-19, es importante tener presente que estamos ante una emergencia sanitaria que ha impactado de múltiples formas a la población mundial. Se ha demostrado que esta enfermedad ha provocado secuelas en diversos órganos y sistemas con un grado de afectación variable. En lo que respecta al área de la audiología, el 
acúfeno siempre ha representado un desafío terapéutico y queda claro que, como consecuencia de la situación actual la incidencia y/o exacerbación de la percepción de este síntoma por múltiples factores es relevante, de tal manera que, en la práctica clínica se añade un evidente reto como parte de las secuelas de COVID-19, por lo que se espera, sea más frecuentemente tratado o diagnosticado y con necesidad de un tratamiento basado en un enfoque multidisciplinario.

\section{Bibliografía}

1. Ruiz-Bravo A, Jimenez-Varela M. SARS-CoV-2 y pandemia de síndrome respiratorio agudo (COVID-19). Ars Pharm. 2020;61(2):63-79.

2. Organización Mundial de la Salud. Preguntas y respuestas sobre la enfermedad por coronavirus (COVID-19). Sitio Web mundial: Organización Mundial de la Salud; 2021 [citado el 4 de septiembre de 2021]. Disponible en: https://www.who.int/es/ emergencies/diseases/novel-coronavirus-2019/advicefor-public/q-a-coronaviruses

3. Valente JP, Pinheiro LA, Carvalho GM, et al. Evaluation of factors related to the tinnitus disturbance. Int Tinnitus J. 2012;17(1):21-25.

4. Curet C, Roitman D. Tinnitus-evaluación y manejo. Rev Med Clin Condes. 2016;27(6):848-862.

5. Martines F, Bentivegna D, Martines E, Sciacca V, Martinciglio G. Characteristics of tinnitus with or without hearing loss: clinical observations in Sicilian tinnitus patients. Auris Nasus Larynx. 2010;37(6):685693.

6. Hoekstra CE, Wesdorp FM, van Zanten GA. Sociodemographic, health, and tinnitus related variables affecting tinnitus severity. Ear Hear. 2014;35(5):544554.

7. National Institute on Deafness and other Communication Disorders. Hoja de información del NIDCD. Tinnitus. Centro de información del NIDCD; 2014 [citado el 28 de agosto de 2021]. Disponible en: https://www.nidcd.nih.gov/sites/ default/files/Documents/health/hearing/es/Tinnitus. pdf

8. American Speech Language Hearing Association. El tinnitus. Serie informativa de audiología: 11566; 2016. [citado el 30 de agosto de 2021]. Disponible en: https://www.asha.org/siteassets/uploadedfiles/ais-eltinnitus.pdf

9. Baguley D, McFerran D, Hall D. Tinnitus. Lancet. 2013;382(9904):1600-1607.

10. Wimmer J, Donoso R, Leiva A, Breinbauer H, Delano P. Tinnitus: Una patología cerebral.
Rev Otorrinolaringol Cirugía Cabeza Cuello. 2019;79(1):125-136. doi: 10.4067/S071848162019000100125.

11. Kim HJ, Lee HJ, An SY, et al. Analysis of the prevalence and associated risk factors of tinnitus in adults. PLoS One. 2015;10(5):e0127578.

12. Schlee W, Hølleland S, Bulla J, et al. The Effect of Environmental Stressors on Tinnitus: A Prospective Longitudinal Study on the Impact of the COVID-19 Pandemic. J Clin Med. 2020;9(9):2756.

13. Beukes EW, Baguley DM, Jacquemin L, et al. Changes in Tinnitus Experiences During the COVID-19 Pandemic. Front Public Health. 2020;8:592878.

14. Xia L, He G, Feng Y, et al. COVID-19 associated anxiety enhances tinnitus. PLoS One. 2021;16(2):e0246328.

15. Anzivino R, Sciancalepore PI, Petrone P, D’Elia A, Petrone D, Quaranta N. Tinnitus revival during COVID-19 lockdown: how to deal with it. Eur Arch Otorhinolaryngol. 2020;278(1):295-296.

16. Micarelli A, Granito I, Carlino P, Micarelli B, Alessandrini M. Self-perceived general and ear-nosethroat symptoms related to the COVID-19 outbreak: a survey study during quarantine in Italy. J Int Med Res. 2020;48(10):300060520961276.

17. Korkmaz MÖ, Eğilmez OK, Özçelik MA, Güven M. Otolaryngological manifestations of hospitalised patients with confirmed COVID-19 infection. Eur Arch Otorhinolaryngol. 2021;278(5):1675-1685.

18. Elibol E. Otolaryngological symptoms in COVID-19. Eur Arch Otorhinolaryngol. 2021;278(4):1233-1236.

19. Munro KJ, Uus K, Almufarrij I, Chaudhuri N, Yioe V. Persistent self-reported changes in hearing and tinnitus in post-hospitalisation COVID-19 cases. Int J Audiol. 2020;59(12):889-890.

20. Viola P, Ralli M, Pisani D, et al. Tinnitus and equilibrium disorders in COVID-19 patients: preliminary results. Eur Arch Otorhinolaryngol. 2020;278(10):3725-3730.

21. Chirakkal P, Al Hail AN, Zada N, Vijayakumar DS. COVID-19 and Tinnitus. Ear Nose Throat J. 2021;100(2_suppl):160S-162S.

22. Kreuzer PM, Landgrebe M, Schecklmann M, Staudinger S, Langguth B. Trauma-associated tinnitus: audiological, demographic and clinical characteristics. PLoS One. 2012;7(9):e45599.

23. Langguth B, Kreuzer PM, Kleinjung T, De Ridder D. Tinnitus: causes and clinical management. Lancet Neurol. 2013;12(9):920-930.

24. Baguley D. Mechanisms of tinnitus. Br Med Bull. 2002;63(1):195-212.

25. Sáez-Jiménez R, Herráiz-Puchol C. Acúfenos: guía clínica en atención primaria. Arch Med Fam. 2006;8(3):190-196.

26. Eggermont JJ. Tinnitus: neurobiological substrates. Drug Discovery Today. 2005;10(19):1283-1290. 